\title{
Increased Metabolic Activity of Indolent Liver Metastases After Resection of a Primary Colorectal Tumor
}

\author{
Marian G.W. Scheer ${ }^{1}$, Thamar H. Stollman ${ }^{1}$, Wouter V. Vogel², Otto C. Boerman², Wim J.G. Oyen ${ }^{2}$, and Theo J.M. Ruers ${ }^{3}$ \\ ${ }^{I}$ Department of Surgery, Radboud University Nijmegen Medical Center, Nijmegen, The Netherlands; ${ }^{2}$ Department of Nuclear \\ Medicine, Radboud University Nijmegen Medical Center, Nijmegen, The Netherlands; and ${ }^{3}$ Department of Surgery, \\ The Netherlands Cancer Institute/Antoni van Leeuwenhoek Hospital, Amsterdam, The Netherlands
}

\begin{abstract}
In murine models, resection of a primary tumor leads to increased vascularization and accelerated growth of metastases that previously had remained microscopic. To study such a potentially inhibitory effect of primary tumors on the outgrowth of distant metastases in humans, we assessed the metabolic activity of liver metastase by ${ }^{18} \mathrm{~F}-\mathrm{FDG}$ PET before and after resection of primary colorectal tumors. Methods: Group A consisted of 8 patients with synchronous colorectal liver metastases who were scheduled for resection of their primary tumor. These patients underwent an ${ }^{18} \mathrm{~F}-\mathrm{FDG}$ PET scan shortly before resection and 2-3 wk after resection of the primary tumor. The patients in a control group (group $\mathrm{B}, n=9$ ) underwent an ${ }^{18} \mathrm{~F}-\mathrm{FDG} \mathrm{PET}$ scan at the time of diagnosis of the liver metastases and a second scan several weeks later, before initiating treatment. There was no surgical intervention between the two ${ }^{18} \mathrm{~F}-\mathrm{FDG}$ PET scans in this group. Results: In group A, the maximum and mean standardized uptake values of the liver metastases clearly increased after resection of the primary tumor, by $38 \% \pm 55 \%$ and $42 \% \pm 52 \%$, respectively, as compared with the first ${ }^{18} \mathrm{~F}$ FDG PET scan. In group $B$, the maximum and mean standardized uptake values of the second ${ }^{18} \mathrm{~F}-\mathrm{FDG}$ PET scan were not significantly higher than those of the first ${ }^{18} \mathrm{~F}-\mathrm{FDG}$ PET scan; $-11 \% \pm$ $23 \%$ and $1 \% \pm 29 \%$, respectively. The difference in standardized uptake value increase between the 2 groups was statistically significant $(P<0.05)$. Conclusion: Our data cannot differentiate between the immunologic sequels caused by the surgical trauma itself and those caused by removal of the primary tumor. The observation itself, however, of increased metabolic activity after surgical resection of the primary tumor may have direct clinical applications and suggests the administration of antiangiogenic therapy after surgery of the primary tumor.
\end{abstract}

Key Words: colorectal cancer; liver metastases; ${ }^{18}$ F-FDG PET

J Nucl Med 2008; 49:887-891

DOI: 10.2967/jnumed.107.048371

Received Nov. 5, 2007; revision accepted Feb. 26, 2008.

For correspondence or reprints contact: Marian G.W. Scheer, Department of Surgery, Radboud University Nijmegen Medical Center, P.O. Box 9101, 6500 HB Nijmegen, The Netherlands.

E-mail: M.Scheer@chir.umcn.nl

COPYRIGHT @ 2008 by the Society of Nuclear Medicine, Inc.
$\mathbf{R}$ ecently, it was reported that the growth rate of liver metastases in patients in whom the primary colorectal tumor had been resected was significantly higher than the growth rate of liver metastases in patients in whom the primary tumor was still in situ $(1,2)$. In addition, immunohistochemical analysis revealed an increased proliferation rate and increased vessel density in the metastases in the absence of the primary tumor. These data suggest that outgrowth of metastatic disease may partly be controlled by the primary tumor (3).

In animal models, primary tumor-mediated inhibition of metastatic vascularization and outgrowth is an established concept in tumor biology. Research in this area was initiated by the observation that in the Lewis lung carcinoma mouse model, resection of the primary tumor led to increased vascularization and accelerated growth of distant metastases that had previously remained microscopic. O'Reilly et al. demonstrated in a murine model that the primary tumor produced the potent antiangiogenic compound angiostatin, which prevented vascularization and thereby growth of metastases (4). More recently, it was demonstrated that irradiation of murine angiostatin-producing primary tumors was followed by subsequent rapid growth of the metastases, suggesting a similar phenomenon $(5,6)$. Moreover, when angiostatin was replaced immediately after regression of the primary tumor, outgrowth of the metastases did not occur (5).

${ }^{18}$ F-FDG PET depicts tumors by identifying cells in the body that have increased glucose uptake and metabolism. ${ }^{18} \mathrm{~F}-\mathrm{FDG}$ is transported into cells analogously to glucose and is converted into ${ }^{18} \mathrm{~F}$-FDG-6-phosphate. This metabolite is trapped in the cell, because it is processed in the glycolytic pathway and hence accumulates preferentially in those cells with enhanced glucose uptake, such as tumor cells. Glucose metabolism can be measured mathematically using the standardized uptake value (SUV), a semiquantitative measure of ${ }^{18} \mathrm{~F}-\mathrm{FDG}$ uptake. It has been demonstrated that the extent of ${ }^{18} \mathrm{~F}-\mathrm{FDG}$ uptake in colorectal cancer metastases correlates with disease aggressiveness 
and prognosis (7). So, metabolic activity of the metastases as measured by ${ }^{18} \mathrm{~F}$-FDG PET before and after resection of the primary tumor may indeed reflect the growth rate of the metastases.

In humans, the possible inhibitory effect of the primary tumor on the proliferation of distant metastases has not been studied in detail. To study the inhibitory effect of the primary tumor on the outgrowth of distant metastases in humans, we assessed the metabolic activity of liver metastases by ${ }^{18} \mathrm{~F}-\mathrm{FDG}$ PET before and after resection of the primary colorectal tumor and compared the results with a control group who underwent 2 serial ${ }^{18}$ F-FDG PET scans without any surgical intervention.

\section{MATERIALS AND METHODS}

\section{Patients}

A total of 21 patients with colorectal liver metastases and no prior chemotherapy during the last 6 mo were potentially eligible for the study. Patients were prospectively included after they had given informed consent. The study was approved by the Institutional Review Board of the Radboud University Nijmegen Medical Centre. The presence of liver metastases was histologically confirmed in 10 patients (mainly patients from group B). In the other 11 patients, the presence of liver metastases was, apart from ${ }^{18}$ F-FDG PET, also determined by ultrasonography $(n=5)$ and CT $(n=7)$. Patients in whom most metastatic lesions showed SUVs greater than 10 at the initial ${ }^{18}$ F-FDG PET scan were excluded from the study $(n=3)$ because it has been recognized that liver metastases with high initial ${ }^{18} \mathrm{~F}-\mathrm{FDG}$ uptake grow quickly and tend to develop central necrosis. The development of these necrotic areas makes serial ${ }^{18} \mathrm{~F}-\mathrm{FDG}$ PET quantification unreliable because of reduced ${ }^{18} \mathrm{~F}$-FDG uptake in partial necrosis and absence of ${ }^{18}$ F-FDG uptake in necrosis. (8) Another patient was excluded because a second liver metastasis developed just adjacent to the first metastasis, making measurement of separate SUVs impossible.

Two groups of patients were analyzed (Table 1). Group A consisted of patients who presented with primary colorectal cancer and synchronous liver metastases, scheduled for resection of the primary tumor $(n=8)$. ${ }^{18} \mathrm{~F}-\mathrm{FDG}$ PET was performed shortly before (mean, $14 \mathrm{~d}$; range, 3-36 d) and 2-3 wk after resection of the colorectal primary tumor. Group B consisted of patients with colorectal liver metastases in whom the primary tumor had been resected at least 6 mo earlier $(n=7)$ or with the primary tumor in situ without being scheduled for primary resection $(n=2)$. In this group, the multidisciplinary team decided to adopt a wait-and-see policy for some time before a definite decision was made to further treat the colorectal liver metastases. In this group, serial ${ }^{18}$ F-FDG PET was performed without any surgical or medical intervention in between. Eventually, patients in group B were scheduled for either hepatic resection $(n=6)$ or further systemic chemotherapy $(n=3)$ after both ${ }^{18}$ F-FDG PET scans.

\section{${ }^{18}$ F-FDG PET}

A dedicated PET/CT scanner (ECAT-EXACT or Biograph PET/ CT; Siemens/CTI) was used for data acquisition. Before ${ }^{18} \mathrm{~F}-\mathrm{FDG}$ injection, patients fasted for at least $6 \mathrm{~h}$. Intake of sugar-free liquids was permitted. One hour after intravenous injection of 200-220 MBq of ${ }^{18}$ F-FDG (Mallinckrodt Medical), emission and transmission images of the area between the upper part of the femora and the base of the skull were acquired. The images were corrected for attenuation and reconstructed using ordered-subsets expectation maximization.

TABLE 1

Results of Group Analysis

\begin{tabular}{|c|c|c|c|}
\hline Characteristic & Group A & Group B & $P$ \\
\hline Mean age $( \pm S D)(y)$ & $62 \pm 13$ & $69 \pm 9$ & NS \\
\hline \multicolumn{4}{|l|}{ Sex } \\
\hline Number of men & $5(62)$ & $7(78)$ & NS \\
\hline Number of women & $3(38)$ & $2(22)$ & \\
\hline \multicolumn{4}{|l|}{ Number of tumors of the following type: } \\
\hline T1 & $0(0)$ & $0(0)$ & NS \\
\hline $\mathrm{T} 2$ & $3(38)$ & $1(14)$ & \\
\hline T3 & $4(50)$ & $6(86)$ & \\
\hline T4 & $1(12)$ & $0(0)$ & \\
\hline NO & $2(25)$ & $2(29)$ & NS \\
\hline $\mathrm{N}+$ & $6(75)$ & $5(71)$ & \\
\hline MO & $0(0)$ & $2(22)$ & $\mathrm{NS}(P=0.51)$ \\
\hline M1 & $8(100)$ & $7(78)$ & \\
\hline \multicolumn{4}{|l|}{ Number of tumors for which the largest metastasis was... } \\
\hline$\leq 2 \mathrm{~cm}$ & $2(25)$ & $4(44)$ & NS \\
\hline $2.1-4.0 \mathrm{~cm}$ & $4(50)$ & $5(56)$ & \\
\hline$>4.0 \mathrm{~cm}$ & $2(25)$ & $0(0)$ & \\
\hline Mean number of metastases $( \pm S D)$ & $6.1 \pm 4.8$ & $2.1 \pm 1.5$ & $P=0.02$ \\
\hline Mean SUVmax on first ${ }^{18}$ F-FDG PET scan $( \pm S D)$ & $7.1 \pm 3.2$ & $6.0 \pm 2.8$ & NS \\
\hline Mean number of days between ${ }^{18} \mathrm{~F}-\mathrm{FDG}$ PET scans $( \pm \mathrm{SD})$ & $35 \pm 14$ & $69 \pm 39$ & NS \\
\hline $\begin{array}{l}\mathrm{NS}=\text { not statistically significant. } \\
\text { Data in parentheses are percentages. }\end{array}$ & & & \\
\hline
\end{tabular}


To determine the metabolic activity of the liver metastases, an experienced nuclear physician who was unaware of the time point of ${ }^{18}$ F-FDG PET drew volumes of interest around all metastases. For this procedure, an automatic 50\% isocontour (ECAT software tool) was used, enclosing pixels with $50 \%$ or more of the maximum radioactivity within the volume of interest. Mean SUV (50\% isocontour) and maximum SUV (SUVmax) were calculated using the concentration of ${ }^{18} \mathrm{~F}-\mathrm{FDG}$ in the volume of interest as measured by PET, divided by the injected dose and multiplied by body weight as a normalization factor. For each patient, the mean SUV and SUVmax were determined by calculating the average value of all liver lesions.

\section{Statistical Analysis}

SUV measurements were analyzed by independent $t$ tests. Differences were considered statistically significant when $P$ was 0.05 or less. Data are presented as mean values \pm SDs.

\section{RESULTS}

Patient data and tumor characteristics are summarized in Table 1. Age, sex, $\mathrm{N}$ stage of the primary tumor, and size of the metastases were similar for both groups. The number of metastases was significantly higher in group B, but initial SUVs, which may reflect possible biologic behavior of the metastases (7), were not statistically different between the 2 groups. On the first ${ }^{18}$ F-FDG PET at study entry, the mean
SUVmax was $7.1 \pm 3.2$ for group $\mathrm{A}$, and $6.0 \pm 2.8$ for group B.

The interval between the two ${ }^{18} \mathrm{~F}-\mathrm{FDG}$ PET scans in patients of group A, who underwent a scan before and after surgery of the primary tumor, was $35 \mathrm{~d}$ (range, 21-57 d). The interval between the two ${ }^{18} \mathrm{~F}-\mathrm{FDG}$ PET scans in patients of group $\mathrm{B}$, who underwent 2 scans without any intervention, was $69 \mathrm{~d}$ (range, 19-126 d).

The SUV was measured for only those metastatic lesions that could clearly be identified as separate. The average SUVmax at baseline and the average SUVmax at follow-up is shown in Table 2 for all patients. As mentioned earlier, patients in whom most of the lesions showed an initial SUVmax of more than 10 were excluded from further analysis. The increase in SUVmax was measured for each lesion, and the average of the changes in SUVmax is shown for each patient. In group A, the SUVmax and mean SUV clearly increased after resection of the primary tumor in 6 of 8 patients (Fig. 1). The mean increase in SUVmax and mean SUV was $38 \% \pm 55 \%$ and $42 \% \pm 52 \%$, respectively. In contrast, in group B none of the patients showed a significant difference between the two ${ }^{18}$ F-FDG PET scans. In this group, the difference in SUVmax and mean SUV was $-11 \% \pm 23 \%$ and $1 \% \pm 29 \%$, respectively. The difference in SUV increase between the 2 groups was statistically

TABLE 2

Data on SUVmax

\begin{tabular}{|c|c|c|c|c|c|}
\hline Group & $\begin{array}{l}\text { Total number } \\
\text { of lesions }\end{array}$ & $\begin{array}{l}\text { Lesions } \\
\text { measured }\end{array}$ & $\begin{array}{c}\text { Average SUVmax } \\
\text { at baseline }\end{array}$ & $\begin{array}{c}\text { Average SUVmax } \\
\text { at follow-up }\end{array}$ & $\begin{array}{c}\text { Average increase } \\
\text { in SUVmax (\%) }\end{array}$ \\
\hline \multicolumn{6}{|l|}{ Group A } \\
\hline 1 & 13 & 2 & 7.1 & 12.1 & 71 \\
\hline 2 & 6 & 5 & 8.8 & 12.8 & 75 \\
\hline 3 & 1 & 1 & 16.7 & 12.3 & -26 \\
\hline 4 & 4 & 3 & 3.8 & 4.4 & 22 \\
\hline 5 & 1 & 1 & 5.3 & 12.8 & 142 \\
\hline 6 & 14 & 6 & 8.7 & 7.0 & -17 \\
\hline 7 & 3 & 3 & 6.2 & 7.6 & 23 \\
\hline 8 & 4 & 4 & 6.3 & 5.3 & -16 \\
\hline 9 & 18 & 6 & 9.9 & 9.9 & 1 \\
\hline 10 & 4 & 3 & 7.2 & 6.2 & 0 \\
\hline Mean increase in SUVmax & & & & & $38 \%$ \\
\hline \multicolumn{6}{|l|}{ Group B } \\
\hline 11 & 3 & 2 & 5.9 & 6.4 & 9 \\
\hline 12 & 3 & 3 & 5.6 & 4.4 & -23 \\
\hline 13 & 1 & 1 & 5.4 & 6.0 & 11 \\
\hline 14 & 4 & 2 & 11.5 & 15.8 & 43 \\
\hline 15 & 6 & 6 & 7.6 & 8.3 & 13 \\
\hline 16 & 1 & 1 & 7.8 & 2.3 & -57 \\
\hline 17 & 2 & 2 & 5.2 & 13.7 & 179 \\
\hline 18 & 1 & 1 & 2.2 & 1.6 & -27 \\
\hline 19 & 1 & 1 & 1.7 & 1.6 & -6 \\
\hline 20 & 1 & 1 & 5.4 & 4.5 & -17 \\
\hline 21 & 2 & 2 & 5.6 & 5.1 & -4 \\
\hline Mean increase in SUVmax & & & & & $-11 \%$ \\
\hline
\end{tabular}

Patients 3, 9, and 14 were excluded from further analyses because initial SUVmax for most metastatic lesions was $>10$. Patient 17 was excluded because second liver metastasis developed just adjacent to first metastasis, making measurement of separate SUVs impossible. 
FIGURE 1. (A) Graph showing major increase in group A SUVmax between ${ }^{18} \mathrm{~F}-\mathrm{FDG}$ PET before (T1) and after (T2) resection of primary colorectal tumor. (B) Graph showing no significant increase in group B SUVmax during T1-to-T2 interval comparable to that for group $A$.

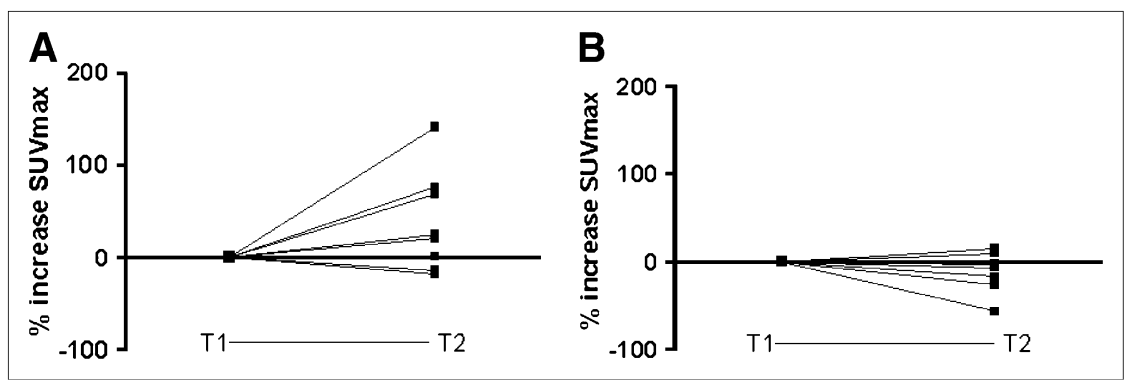

significant for both SUVmax $(P=0.027)$ and mean SUV $(P=0.048)$. A typical example of increased ${ }^{18} \mathrm{~F}-\mathrm{FDG}$ uptake in liver metastases after resection of the primary tumor, as compared with ${ }^{18} \mathrm{~F}$-FDG uptake in 2 subsequent ${ }^{18}$ F-FDG PET scans in a patient of group $\mathrm{B}$, is shown in Figure 2. No correlation was found when comparing the change in SUVmax with the change in diameter of the lesions. The mean increase in diameter in group A was $9.3 \%$, with an increase in SUVmax of 38\%. In contrast, in group B the mean diameter of the metastases showed an increase of $58 \%$, with a decrease in the SUVmax of $11 \%$.

\section{DISCUSSION}

Although only a limited number of patients could be included, our results demonstrated a significant increase in ${ }^{18}$ F-FDG uptake of colorectal liver metastases after resection of the primary tumor had been observed. In contrast, ${ }^{18} \mathrm{~F}-$ FDG uptake in liver metastases remained stable on 2 subsequent ${ }^{18} \mathrm{~F}$-FDG PET scans of patients without any surgical or other therapeutic intervention between the scans.

These results may indicate that in humans, as in animal models, the primary tumor can inhibit growth of its metastases. This inhibitory effect on secondary tumor growth is reversed when the primary tumor is resected. For example, in mice, $\mathrm{Li}$ et al. demonstrated increased microvessel density, a higher cell proliferation index of tumor cells, and a decreased apoptotic index after resection of a primary tumor, compared with a sham operation (9).

On the other hand, the increased metabolic activity observed after resection of the primary colorectal tumor may also be caused by the surgical trauma of the resection itself. Surgery alone might stimulate proinflammatory cytokines (IL-6 and IL-1 $\beta$ ), resulting in enhanced expression of vascular endothelial growth factor and angiogenesis (10). Our data, as presented in this study, cannot differentiate between the 2 hypotheses. Another explanation for the outgrowth of the metastases could be that the increase in ${ }^{18} \mathrm{~F}-\mathrm{FDG}$ uptake is caused by mere time-dependent progression and that synchronous liver metastases (group A) would grow faster than metachronous liver metastases (most patients in group B). This explanation, however, is unlikely because initial SUVs in both groups were identical and the interval between the 2 serial ${ }^{18}$ F-FDG PET scans was even longer in group B than in group A. Furthermore, increased retention of ${ }^{18} \mathrm{~F}$-FDG may be due to an increased size of the lesion; however, our results did not show a correlation between the alteration in tumor size and the change in SUV.

Increased growth of metastases after the removal of a primary tumor has been observed in various animal models and was related to loss of antiangiogenic factors produced by the primary tumor $(4,11)$. The balance between proand antiangiogenic factors within the vascular bed of the

FIGURE 2. (A) ${ }^{18} \mathrm{~F}-\mathrm{FDG}$ PET scan of patient in group A. Rapid outgrowth of indolent liver metastases occurred after removal of primary colorectal carcinoma. SUVmax of largest lesion was 7.0 on first scan and 12.1 on second scan. (B) ${ }^{18} \mathrm{~F}$ FDG PET scan of patient in group B. ${ }^{18} \mathrm{~F}-$ FDG uptake increased slightly between the 2 subsequent PET/CT scans. SUVmax was 7.6 on first scan and 8.3 on second scan.
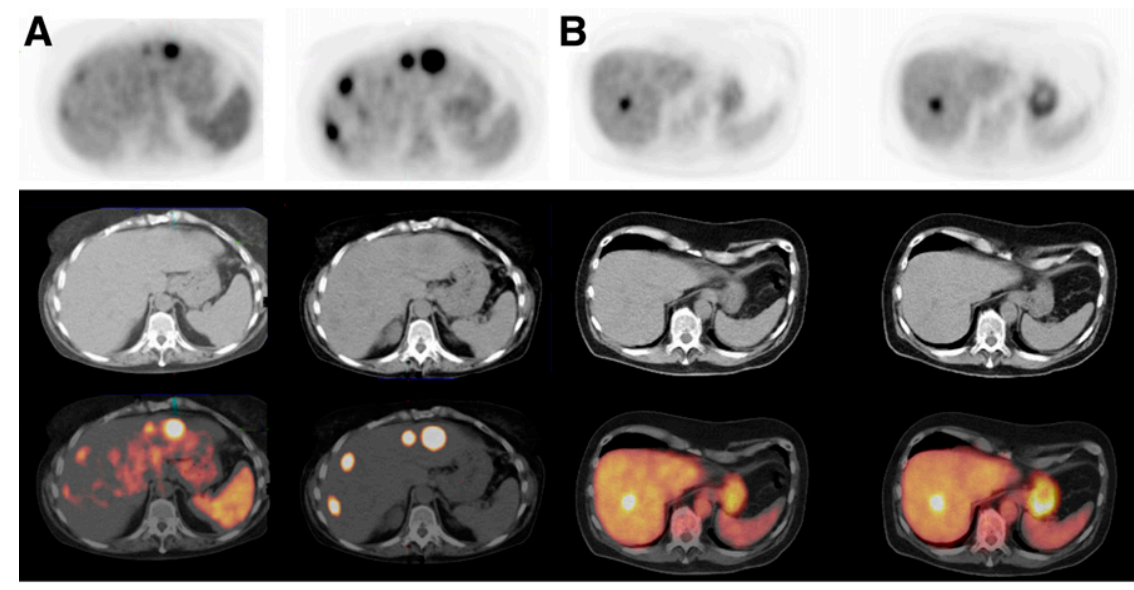

First scan

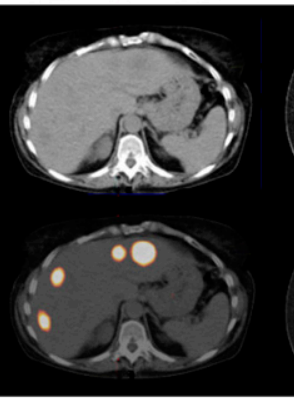

Second scan

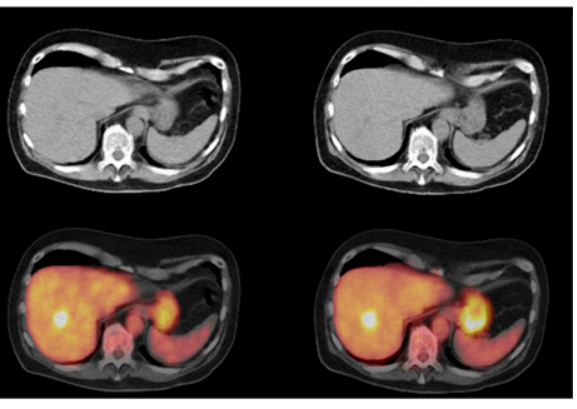

First scan

Second scan 
metastases determines whether the metastases will remain dormant or will proliferate. The dormant state of metastases has been explained by the production of antiangiogenic factors by the primary tumor, such as angiostatin, resulting in inhibition of growth of distant metastases (4). Resection of the primary tumor would interfere with this inhibitory control mechanism, resulting in activation of the angiogenic switch in the metastatic lesions. In patients, this mechanism is supported by earlier findings that vascularization of colorectal liver metastases was significantly increased in the absence of the primary tumor (1). However, data on angiostatin production in patients with colorectal cancer are scarce and indicate at the least that not all primary colorectal tumors may produce angiostatin (12). This indication could also explain why 2 patients in our series did not show an increase in metabolic activity after resection of the primary tumor.

In tumor-bearing animals, vascularization and subsequent growth of distant metastases can be slowed by systemic administration of angiogenesis inhibitors (13-15).

Although the precise mechanism of increased metabolic activity after resection of the primary colorectal tumor as observed in our study still has to be elucidated, slowing of this accelerated growth by angiogenesis inhibitors seems promising. The present approach of serial ${ }^{18} \mathrm{~F}-\mathrm{FDG}$ PET scans before and after resection of the primary tumor would possibly allow a direct measurement of the therapeutic effect of such antiangiogenic therapy. Such studies might provide insight into the use of adjuvant antiangiogenic agents to prevent accelerated outgrowth of distant metastases after resection of the primary colorectal tumor.

\section{REFERENCES}

1. Peeters CF, Westphal JR, de Waal RM, Ruiter DJ, Wobbes T, Ruers TJ. Vascular density in colorectal liver metastases increases after removal of the primary tumor in human cancer patients. Int J Cancer. 2004;112:554-559.

2. Simpson-Herren L, Sanford AH, Holmquist JP. Effects of surgery on the cell kinetics of residual tumor. Cancer Treat Rep. 1976;60:1749-1760.

3. Peeters CF, de Waal RM, Wobbes T, Westphal JR, Ruers TJ. Outgrowth of human liver metastases after resection of the primary colorectal tumor: a shift in the balance between apoptosis and proliferation. Int J Cancer. 2006;119:1249-1253.

4. O'Reilly MS, Holmgren L, Shing Y, et al. Angiostatin: a novel angiogenesis inhibitor that mediates the suppression of metastases by a Lewis lung carcinoma. Cell. 1994;79:315-328.

5. Camphausen K, Moses MA, Beecken WD, Khan MK, Folkman J, O'Reilly MS. Radiation therapy to a primary tumor accelerates metastatic growth in mice. Cancer Res. 2001;61:2207-2211.

6. von Essen CF. Radiation enhancement of metastasis: a review. Clin Exp Metastasis. 1991;9:77-104.

7. de Geus-Oei LF, Wiering B, Krabbe PF, Ruers TJ, Punt CJ, Oyen WJ. FDG-PET for prediction of survival of patients with metastatic colorectal carcinoma. Ann Oncol. 2006;17:1650-1655.

8. Miller FH, Keppke AL, Reddy D, et al. Response of liver metastases after treatment with yttrium-90 microspheres: role of size, necrosis, and PET. AJR. 2007;188:776-783.

9. Li TS, Kaneda Y, Ueda K, Hamano K, Zempo N, Esato K. The influence of tumour resection on angiostatin levels and tumour growth: an experimental study in tumour-bearing mice. Eur J Cancer. 2001;37:2283-2288.

10. Nagengast WB, de Vries EG, Hospers GA, et al. In vivo VEGF imaging with radiolabeled bevacizumab in a human ovarian tumor xenograft. J Nucl Med. 2007;48:1313-1319.

11. Holmgren L, O'Reilly MS, Folkman J. Dormancy of micrometastases: balanced proliferation and apoptosis in the presence of angiogenesis suppression. Nat Med. 1995;1:149-153.

12. Sten-Linder M, Linder C, Strander H, et al. Angiostatin fragments in urine from patients with malignant disease. Anticancer Res. 1999;19:3409-3414.

13. O'Reilly MS, Holmgren L, Chen C, Folkman J. Angiostatin induces and sustains dormancy of human primary tumors in mice. Nat Med. 1996;2:689-692.

14. Wu Z, O'Reilly MS, Folkman J, Shing Y. Suppression of tumor growth with recombinant murine angiostatin. Biochem Biophys Res Commun. 1997;236:651-654.

15. Bergers G, Javaherian K, Lo KM, Folkman J, Hanahan D. Effects of angiogenesis inhibitors on multistage carcinogenesis in mice. Science. 1999;284:808-812. 